International Mathematical Forum, Vol. 8, 2013, no. 29, 1405 - 1412 HIKARI Ltd, www.m-hikari.com

http://dx.doi.org/10.12988/imf.2013.37144

\title{
On a Principal Ideal Domain that is not a Euclidean Domain
}

\author{
Conan Wong \\ Department of Mathematics \\ University of British Columbia, Vancouver, Canada \\ conan@math.ubc.ca
}

Copyright (C) 2013 Conan Wong. This is an open access article distributed under the Creative Commons Attribution License, which permits unrestricted use, distribution, and reproduction in any medium, provided the original work is properly cited.

\begin{abstract}
The ring $\mathbb{Z}\left[\frac{1+\sqrt{-19}}{2}\right]$ is usually given as a first example of a principal ideal domain (PID) that is not a Euclidean domain. This paper gives an elementary and more direct proof that $\mathbb{Z}\left[\frac{1+\sqrt{-19}}{2}\right]$ is indeed a PID.
\end{abstract}

Mathematics Subject Classification: 11R04, 13F07, 13F10

Keywords: Euclidean domain, principal ideal domain, quadratic integer ring

\section{Introduction}

In a course on abstract algebra, one proves that all Euclidean domains are principal ideal domains (PIDs). The ring $\mathbb{Z}\left[\frac{1+\sqrt{-19}}{2}\right]$ is then usually given as a "simple" example of a PID that is not a Euclidean domain. However, details of this example are usually omitted. Some textbooks leave it as a series of exercises for the student. There have been efforts to simplify the proof that $\mathbb{Z}\left[\frac{1+\sqrt{-19}}{2}\right]$ is indeed a PID but not a Euclidean domain, such as [6], [5] and, most recently, [2]. A comparative survey of the various papers can be found in [3]. 
For ease of notation, let $\omega=\frac{1+\sqrt{-19}}{2}$ henceforth.

It is straightforward to show that $\mathbb{Z}[\omega]$ in not Euclidean and this paper includes an existing proof for completeness. However, the proof that $\mathbb{Z}[\omega]$ is a PID is slightly more difficult. For example, the proofs in [6] and [3] leverage on a theorem due to Dedekind and Hasse, and the ensuing proof requires a breakdown into 5 cases, each corresponding to different elements of $\mathbb{Z}[\omega]$. The proof in [2] is a simplification, intended to make the material more accessible to mathematics students. However, it still requires a partitioning of $\mathbb{Z}[\omega]$ into 7 cases.

This paper provides an elementary and more direct proof that $\mathbb{Z}[\omega]$ is a PID. It is written with the same motivation as [2], utilising only introductory abstract algebra and the absolute value of a complex number, to improve access to comprehension. By partitioning $\mathbb{Z}[\omega]$ differently, the proof in this paper requires a breakdown into only 3 cases.

\section{$2 \mathbb{Z}[\omega]$ is not a Euclidean Domain}

This proof that $\mathbb{Z}[\omega]$ is not a Euclidean domain is similar to the proof in $[2]$ and, as mentioned earlier, is included here for completeness.

Firstly, note that $\omega^{2}=\omega-5$. Thus, $\mathbb{Z}[\omega]=\{a+b \omega \mid a, b \in \mathbb{Z}\}$. Also, as the minimal polynomial of $\omega$ over $\mathbb{Z}$ is $x^{2}-x+5$, which is Eisenstein and hence irreducible, $\mathbb{Z}[\omega]$ is an integral domain. For any element $\alpha \in \mathbb{Z}[\omega] \subset \mathbb{C}$, we have the usual absolute value $|\alpha|=\alpha \bar{\alpha}$, where $\bar{\alpha}$ denotes the usual complex conjugate of $\alpha$. It is easy to see that for any $\alpha \in \mathbb{Z}[\omega], \bar{\alpha} \in \mathbb{Z}[\omega]$ as well. We begin by proving some useful properties relating to the absolute values of elements in $\mathbb{Z}[\omega]$.

Lemma 2.1. For $\alpha \in \mathbb{Z}[\omega] \backslash 0,|\alpha| \in \mathbb{N}$.

Proof. As $\alpha=a+b \omega$ for some $a, b \in \mathbb{Z}$,

$$
|\alpha|=\left[a+b\left(\frac{1+\sqrt{-19}}{2}\right)\right]\left[a+b\left(\frac{1-\sqrt{-19}}{2}\right)\right]=a^{2}+a b+5 b^{2} \in \mathbb{Z}^{\geq 0} .
$$

Since $\alpha \neq 0,|\alpha| \neq 0$. Thus, $|\alpha| \in \mathbb{N}$.

Lemma 2.2. For $\alpha \in \mathbb{Z}[\omega]$, the following statements are equivalent:

(i) $\alpha=-1$ or 1 .

(ii) $\alpha$ is a unit in $\mathbb{Z}[\omega]$.

(iii) $|\alpha|=1$. 
Proof. (i) $\Rightarrow$ (ii) is clear.

For (ii) $\Rightarrow$ (iii), if $\alpha$ is a unit in $\mathbb{Z}[\omega]$, then $\exists \beta \in \mathbb{Z}[\omega]$ such that $\alpha \beta=1$. Then $1=|\alpha \beta|=|\alpha||\beta|$. By Lemma 2.1, we must have $|\alpha|=|\beta|=1$.

For (iii) $\Rightarrow$ (i), we write $\alpha=a+b \omega$ for some $a, b \in \mathbb{Z}$. Then $1=|\alpha|=$ $a^{2}+a b+5 b^{2}=\left(a+\frac{b}{2}\right)^{2}+\frac{19}{4} b^{2}$. As $a, b \in \mathbb{Z}$, we must have $b=0$, which in turn implies that $a^{2}=1$.

Our proof that $\mathbb{Z}[\omega]$ is not Euclidean features some "special elements" of $\mathbb{Z}[\omega]$, namely $\pm 1, \pm 2$ and \pm 3 . Lemma 2.2 showed that \pm 1 are the only units in $\mathbb{Z}[\omega]$. The following lemma shows that \pm 2 and \pm 3 are irreducible in $\mathbb{Z}[\omega]$. Recall that an element of a ring is irreducible if it satisfies the following properties:

(i) It is a nonzero non-unit in the ring; and

(ii) If it is written as a product of 2 elements of the ring, exactly 1 of them is a unit.

Lemma 2.3. \pm 2 and \pm 3 are irreducible in $\mathbb{Z}[\omega]$.

Proof. As \pm 1 are units, it suffices to prove that 2 and 3 are irreducible.

2 is clearly a nonzero non-unit in $\mathbb{Z}[\omega]$, since $\frac{1}{2} \notin \mathbb{Z}[\omega]$. Suppose we write $2=\alpha \beta$ for some $\alpha, \beta \in \mathbb{Z}[\omega]$. Then $4=|2|=|\alpha||\beta|$. By Lemma 2.1, this implies that $(|\alpha|,|\beta|)=(1,4),(2,2)$ or $(4,1)$. By Lemma 2.2, the first and the last cases would imply that either $\alpha$ or $\beta$ is a unit respectively and, hence, 2 is irreducible.

For the case $(|\alpha|,|\beta|)=(2,2)$, writing $\alpha=a+b \omega$ for some $a, b \in \mathbb{Z}$, we would get $2=|\alpha|=a^{2}+a b+5 b^{2}=\left(a+\frac{b}{2}\right)^{2}+\frac{19}{4} b^{2}$. But then $a, b \in \mathbb{Z}$ means that $b=0$, which in turn implies that $a^{2}=2$, a contradiction.

The proof that 3 is irreducible is similar.

Theorem 2.4. $\mathbb{Z}[\omega]$ is not a Euclidean domain.

Proof. Assume the contrary, i.e. that $\mathbb{Z}[\omega]$ is a Euclidean domain. Then there exists a Euclidean degree function $D: \mathbb{Z}[\omega] \backslash 0 \rightarrow \mathbb{N}$ satisfying the Euclidean Division Algorithm:

For $\alpha, \beta \in \mathbb{Z}[\omega]$ where $\beta \neq 0$, there exist $q, r \in \mathbb{Z}[\omega]$ such that $\alpha=\beta q+r$ and either $r=0$ or $D(r)<D(\beta)$.

As the range of $D$ is $\mathbb{N}$, we can choose $m \in \mathbb{Z}[\omega]$ such that $D(m)$ is as small as possible subject to $m$ not being zero or a unit. Then let $q, r \in \mathbb{Z}[\omega]$ be the quotient and remainder, respectively, when we divide 2 by $m$ in $\mathbb{Z}[\omega]$, i.e. 
$2=m q+r, \quad$ where $r=0$ or $D(r)<D(m)$.

$D(m)$ is already as small as possible subject to $m$ being a nonzero non-unit. So either $r=0$, or else $D(r)<D(m)$ implies that $r$ is a unit in $\mathbb{Z}[\omega]$, i.e. $r=-1$ or 1 (by Lemma 2.2).

If $r=0$, then $m$ divides 2. Since $m$ is not a unit and 2 is irreducible in $\mathbb{Z}[\omega]$ (by Lemma 2.3), this means that $m=-2$ or 2 . (Again, we have used the fact that the only units in $\mathbb{Z}[\omega]$ are -1 and 1.)

If $r=-1$, then $m$ divides 3. By a similar line of reasoning as in the case above, $m=-3$ or 3 .

If $r=1$, then $m$ divides 1 , which is a contradiction since $m$ is not a unit by assumption.

Thus, we have shown that the possible choices for $m$ (i.e. the nonzero nonunit elements of $\mathbb{Z}[\omega]$ with minimal degree $D)$ are \pm 2 and \pm 3 .

Next, we divide $\omega$ by $m$ in $\mathbb{Z}[\omega]$, getting

$$
\omega=m q^{\prime}+r^{\prime}, \quad \text { for some } q^{\prime}, r^{\prime} \in \mathbb{Z}[\omega] \text { where } r^{\prime}=0 \text { or } D\left(r^{\prime}\right)<D(m) .
$$

By the same argument as above, this implies that $r^{\prime}=-1,0$ or 1 .

If $r^{\prime}=-1$, then $m$ divides $1+\omega$ in $\mathbb{Z}[\omega]$. But as $m \in\{ \pm 2, \pm 3\}, \frac{1}{m}(1+\omega) \notin$ $\mathbb{Z}[\omega]$, a contradiction.

If $r^{\prime}=0$, then $m$ divides $\omega$ in $\mathbb{Z}[\omega]$. But as $m \in\{ \pm 2, \pm 3\}, \frac{1}{m}(\omega) \notin \mathbb{Z}[\omega]$, a contradiction.

If $r^{\prime}=1$, then $m$ divides $-1+\omega$ in $\mathbb{Z}[\omega]$. But as $m \in\{ \pm 2, \pm 3\}, \frac{1}{m}(-1+\omega) \notin$ $\mathbb{Z}[\omega]$, a contradiction.

\section{$3 \mathbb{Z}[\omega]$ is a Principal Ideal Domain}

This proof is based on a combination of ideas from [1] and [7]. Importantly, it hinges on the absolute values of elements in $\mathbb{Z}[\omega]$ and, thus, uses Lemma 2.1 from the previous section.

Theorem 3.1. $\mathbb{Z}[\omega]$ is a principal ideal domain.

Proof. Let $I$ be any nonzero ideal in $\mathbb{Z}[\omega]$. As Lemma 2.1 showed that the absolute values of nonzero elements in $\mathbb{Z}[\omega]$ are natural numbers, we can pick a nonzero $\beta \in I$ such that $|\beta|$ is as small as possible among the nonzero elements of $I$. We seek to show that $I=(\beta)$, i.e. $I$ is a principal ideal generated by $\beta$. 
Assume the contrary. Then there exists a nonzero $\alpha \in I \backslash(\beta)$. Consider $\frac{\alpha}{\beta} \in \mathbb{C}$. As $\omega=\frac{1}{2}+\frac{\sqrt{19}}{2} i \in \mathbb{C}$, we can pick $m \in \mathbb{Z}$ such that

$$
-\frac{\sqrt{19}}{4}<\operatorname{Im}\left(\frac{\alpha}{\beta}+m \omega\right) \leq \frac{\sqrt{19}}{4}
$$

where $I m$ refers to the imaginary part of a complex number. We now split up the argument into 2 cases, depending on the value of $\operatorname{Im}\left(\frac{\alpha}{\beta}+m \omega\right)$.

$\underline{\text { Case } 1 .}-\frac{\sqrt{3}}{2}<\operatorname{Im}\left(\frac{\alpha}{\beta}+m \omega\right)<\frac{\sqrt{3}}{2}$

In this more straightforward case, we can pick $n \in \mathbb{Z}$ such that

$$
-\frac{1}{2}<\operatorname{Re}\left(\frac{\alpha}{\beta}+m \omega+n\right) \leq \frac{1}{2}
$$

where Re refers to the real part of a complex number. Since $\operatorname{Im}\left(\frac{\alpha}{\beta}+m \omega+n\right)=$ $\operatorname{Im}\left(\frac{\alpha}{\beta}+m \omega\right)$, we also have

$$
-\frac{\sqrt{3}}{2}<\operatorname{Im}\left(\frac{\alpha}{\beta}+m \omega+n\right)<\frac{\sqrt{3}}{2} .
$$

Thus, $\left|\frac{\alpha}{\beta}+m \omega+n\right|<\left(\frac{1}{2}\right)^{2}+\left(\frac{\sqrt{3}}{2}\right)^{2}=1$, and $|\alpha+(m \omega+n) \beta|=\left|\frac{\alpha}{\beta}+m \omega+n\right||\beta|<$ $|\beta|$.

But as $\alpha, \beta \in I$ and $m \omega+n \in \mathbb{Z}[\omega]$, it follows that $\alpha+(m \omega+n) \beta \in I$. Since $|\beta|$ is as small as possible among the absolute values of nonzero elements in $I,|\alpha+(m \omega+n) \beta|<|\beta|$ implies that $\alpha+(m \omega+n) \beta=0$. Thus, $\alpha \in(\beta)$, which contradicts our assumption.

Case 2. Either $-\frac{\sqrt{19}}{4}<\operatorname{Im}\left(\frac{\alpha}{\beta}+m \omega\right) \leq-\frac{\sqrt{3}}{2}$, or $\frac{\sqrt{3}}{2} \leq \operatorname{Im}\left(\frac{\alpha}{\beta}+m \omega\right) \leq \frac{\sqrt{19}}{4}$

If $-\frac{\sqrt{19}}{4}<\operatorname{Im}\left(\frac{\alpha}{\beta}+m \omega\right) \leq-\frac{\sqrt{3}}{2}$, then let $\alpha^{\prime}=-\alpha-m \omega \beta$.

If $\frac{\sqrt{3}}{2} \leq \operatorname{Im}\left(\frac{\alpha}{\beta}+m \omega\right) \leq \frac{\sqrt{19}}{4}$, then let $\alpha^{\prime}=\alpha+m \omega \beta$.

In both instances, since $\alpha, \beta \in I$ and $m, \omega \in \mathbb{Z}[\omega]$, we see that $\alpha^{\prime} \in I$. But if $\alpha^{\prime} \in(\beta)$, then $\alpha=\mp\left(\alpha^{\prime}-m \omega \beta\right) \in(\beta)$ as well, which contradicts our assumption that $\alpha \notin(\beta)$. Thus, in both instances, we have found an element $\alpha^{\prime} \in I \backslash(\beta)$ such that

$$
\frac{\sqrt{3}}{2} \leq \operatorname{Im}\left(\frac{\alpha^{\prime}}{\beta}\right) \leq \frac{\sqrt{19}}{4}
$$

Now, as in Case 1 , we can find $n \in \mathbb{Z}$ such that 


$$
-\frac{1}{2}<\operatorname{Re}\left(\frac{\alpha^{\prime}}{\beta}+n\right) \leq \frac{1}{2} .
$$

Let $\alpha^{\prime \prime}=\alpha^{\prime}+n \beta \in I$. Note that $\operatorname{Im}\left(\frac{\alpha^{\prime \prime}}{\beta}\right)=\operatorname{Im}\left(\frac{\alpha^{\prime}}{\beta}\right)$. As before, if $\alpha^{\prime \prime} \in(\beta)$, then $\alpha^{\prime}=\alpha^{\prime \prime}-n \beta \in(\beta)$ as well, which is a contradiction. Thus, we have found an element $\alpha^{\prime \prime} \in I \backslash(\beta)$ such that

$$
\frac{\sqrt{3}}{2} \leq \operatorname{Im}\left(\frac{\alpha^{\prime \prime}}{\beta}\right) \leq \frac{\sqrt{19}}{4} \text {, and }-\frac{1}{2}<\operatorname{Re}\left(\frac{\alpha^{\prime \prime}}{\beta}\right) \leq \frac{1}{2} .
$$

To finish the proof, we consider the element $\frac{2 \alpha^{\prime \prime}}{\beta}-\omega \in \mathbb{C}$, which will give us the desired contradictions via 2 subcases. Since $\omega=\frac{1}{2}+\frac{\sqrt{19}}{2} i$, we get that

$$
-\frac{3}{2}<\operatorname{Re}\left(\frac{2 \alpha^{\prime \prime}}{\beta}-\omega\right) \leq \frac{1}{2} .
$$

Noting that $\sqrt{19}<\sqrt{27}=3 \sqrt{3}$, we get $\sqrt{3}-\frac{\sqrt{19}}{2}>\sqrt{3}-\frac{3 \sqrt{3}}{2}=-\frac{\sqrt{3}}{2}$. Thus,

$$
-\frac{\sqrt{3}}{2}<\sqrt{3}-\frac{\sqrt{19}}{2} \leq \operatorname{Im}\left(\frac{2 \alpha^{\prime \prime}}{\beta}-\omega\right) \leq 0 .
$$

Case $2(\mathrm{a}) .-\frac{1}{2}<\operatorname{Re}\left(\frac{2 \alpha^{\prime \prime}}{\beta}-\omega\right) \leq \frac{1}{2}$

In this sub-case, since $\left|\frac{2 \alpha^{\prime \prime}}{\beta}-\omega\right|<\left(\frac{1}{2}\right)^{2}+\left(-\frac{\sqrt{3}}{2}\right)^{2}=1$, we see that $\left|2 \alpha^{\prime \prime}-\omega \beta\right|=$ $\left|\frac{2 \alpha^{\prime \prime}}{\beta}-\omega\right||\beta|<|\beta|$. Since $\alpha^{\prime \prime}, \beta \in I$, it follows that $2 \alpha^{\prime \prime}-\omega \beta \in I$ as well. But as $|\beta|$ is as small as possible among the absolute values of nonzero elements in $I,\left|2 \alpha^{\prime \prime}-\omega \beta\right|<|\beta|$ implies that $2 \alpha^{\prime \prime}-\omega \beta=0$. This means that $\frac{\omega \beta}{2}=\alpha^{\prime \prime} \in I$.

Now as $\bar{\omega} \in \mathbb{Z}[\omega]$ and $\bar{\omega} \omega=5$, we have $\frac{5}{2} \beta=\bar{\omega}\left(\frac{\omega \beta}{2}\right) \in I$. And since $\beta \in I$, we see that $\frac{1}{2} \beta=\frac{5}{2} \beta-2 \beta \in I$ as well. But then $0<\left|\frac{1}{2} \beta\right|=\frac{1}{4}|\beta|<|\beta|$ contradicts the minimality of $|\beta|$ among the absolute values of nonzero elements in $I$, which completes the proof of this sub-case.

Case $2(\mathrm{~b}) .-\frac{3}{2}<\operatorname{Re}\left(\frac{2 \alpha^{\prime \prime}}{\beta}-\omega\right) \leq-\frac{1}{2}$

In this sub-case, we "shift by 1 " to get a proof similar to Case 2(a), i.e. we consider $\frac{2 \alpha^{\prime \prime}}{\beta}-\omega+1 \in \mathbb{C}$. Clearly,

$$
-\frac{1}{2}<\operatorname{Re}\left(\frac{2 \alpha^{\prime \prime}}{\beta}-\omega+1\right) \leq \frac{1}{2} \text {, and }-\frac{\sqrt{3}}{2}<\operatorname{Im}\left(\frac{2 \alpha^{\prime \prime}}{\beta}-\omega+1\right) \leq 0
$$

since $\operatorname{Im}\left(\frac{2 \alpha^{\prime \prime}}{\beta}-\omega+1\right)=\operatorname{Im}\left(\frac{2 \alpha^{\prime \prime}}{\beta}-\omega\right)$.

Thus, $\left|\frac{2 \alpha^{\prime \prime}}{\beta}-\omega+1\right|<\left(\frac{1}{2}\right)^{2}+\left(-\frac{\sqrt{3}}{2}\right)^{2}=1$, and we see that $\left|2 \alpha^{\prime \prime}-\omega \beta+\beta\right|=$ $\left|\frac{2 \alpha^{\prime \prime}}{\beta}-\omega+1\right||\beta|<|\beta|$. Since $\alpha^{\prime \prime}, \beta \in I$, it follows that $2 \alpha^{\prime \prime}-\omega \beta+\beta \in I$ as 
well. But as $|\beta|$ is as small as possible among the absolute values of nonzero elements in $I,\left|2 \alpha^{\prime \prime}-\omega \beta+\beta\right|<|\beta|$ implies that $2 \alpha^{\prime \prime}-\omega \beta+\beta=0$. This means that $\frac{\omega-1}{2} \beta=\alpha^{\prime \prime} \in I$.

Now as $\overline{\omega-1} \in \mathbb{Z}[\omega]$ and $(\overline{\omega-1})(\omega-1)=5$, we have $\frac{5}{2} \beta=(\overline{\omega-1})\left(\frac{\omega-1}{2} \beta\right) \in$ $I$. By an argument identical to that in Case $2(\mathrm{a}), \frac{1}{2} \beta \in I$ as well, contradicting the minimality of $|\beta|$ among the absolute values of nonzero elements in $I$ and completing the proof.

\section{Concluding Remarks}

The ring $\mathbb{Z}[\omega]$ is an example of a quadratic integer ring. In general, for a square-free integer $D$, let

$$
\theta=\left\{\begin{array}{lrr}
\sqrt{D} & \text { if } D \equiv 2,3 \quad(\bmod 4) \\
\frac{1+\sqrt{D}}{2} & \text { if } D \equiv 1 \quad(\bmod 4)
\end{array}\right.
$$

Then, $\mathbb{Z}[\theta]$ is a quadratic integer ring (the ring of integers in the quadratic number field, $\mathbb{Q}(\sqrt{D}))$.

It is known that $\mathbb{Z}[\theta]$ is a PID but not a Euclidean domain exactly when $D=-19,-43,-67$ or -163 (see [3], [4] and [5]). This paper dealt with the case $D=-19$. Perhaps a possible next step would be to find a unifying proof (for all 4 cases) that is equally accessible to students in mathematics.

\section{References}

[1] G.M. Bergman, A principal ideal domain that is not Euclidean. [George M. Bergman's website, accessed on 21 January 2013] Available at: math.berkeley.edu/ gbergman/grad.hndts/nonEucPID.ps

[2] O.A. Campoli, A principal ideal domain that is not a Euclidean domain, American Mathematical Monthly, 95 (1988), no. 9, 868-871.

[3] V. Peric and M. Vukovic, Some examples of principal ideal domain which are not Euclidean and some other counterexamples, Novi Sad Journal of Mathematics, 38 (2008), no. 1, 137-154.

[4] H.M. Stark, A complete determination of the complex quadratic fields of class-number one, Michigan Mathematical Journal, 14 (1967), no. 1, 1-27. 
[5] K.S. Williams, Note on non-Euclidean principal ideal domains, Mathematics Magazine, 48 (1975), no. 3, 176-177.

[6] J.C. Wilson, A principal ideal ring that is not a Euclidean ring, Mathematics Magazine, 46 (1973), no. 1, 34-38.

[7] R.A. Wilson, An example of a PID which is not a Euclidean domain. [Robert A. Wilson's website, accessed on 21 January 2013] Available at: www.maths.qmul.ac.uk/ raw/MTH5100/PIDnotED.pdf

\section{Received: July 17, 2013}

